\title{
Zweckentfremdung als Mittelaneignung
}

\author{
Fünf Thesen und eine Schicksalsfrage
}

\begin{abstract}
Das Kugellager fordert entweder ein neues politisches Bewußtsein, das die Werkzeuge der Gesellschaft in Maßen hält, oder es beschwört techno-faschistische Diktatur herauf.

- Ivan Illich (1978b, 111)
\end{abstract}

Längst hatten die Kirchenväter, besonders die griechischen Theologen, die These aufgestellt, Gott habe nach Adams Sündenfall das Paradies keineswegs zerstört. Er habe es nur weggerückt auf die ,Gegenerde', in einen den Menschen nicht erreichbaren Raum. Diese ,Gegenerde‘ aber solle nach der mythischen Theologie jenseits des Ozeans gelegen sein, also hinter einer den Irdischen undurchdringlichen Zone. Nun aber, da die Kühnheit der Entdecker diesen bisher undurchdringlichen Ozean durchfahren und die Hemisphäre der anderen Sterne erreicht - könnte der alte Traum der Menschheit sich nicht doch erfüllen und das Paradies wiedergewonnen werden?

- Stefan Zweig, Amerigo (1944)

\section{Paradies}

Der vorliegende Band ist unserem Umgang mit technischen Artefakten in der gegenwärtigen Welt gewidmet, für welchen insbesondere charakteristisch ist, dass wir als Konsumenten diese Artefakte in der Regel fertig vorfinden und bei Verschleiß entweder wegwerfen oder einem Experten zur Reparatur übergeben. Daher mag es überraschen, wenn ich mit einem Rückblick in die Werkzeugentwicklung in der Vorgeschichte beginne, also in einer Epoche, in welcher die Menschen nur sehr wenig fertig vorfanden und insbesondere noch die Rollen von Konsumenten und Produzenten nahezu aller ihrer Alltagsobjekte in einer Person vereinten.

Meine erste These lautet aber, dass diese beiden Situationen nicht so unterschiedlich sind, sondern sich die beiden Extreme der historischen Entwicklung in einem Punkt wieder zu treffen scheinen. Auch die frühen Menschen finden fertige Dinge vor, nur eben Naturgegenstände, die sie sich sodann allmählich aneignen. Aber auch die Naturgegenstände - die Rohstoffe -, sind ,Black Boxes‘, mit dem einzigen Unterschied, dass sie nicht von Apple mit handelsunüblichen Spezialschrauben verschlossen, sondern - was sich in theologischem Vokabular viel leichter sagen lässt, weshalb ich diese Perspektive provisorisch einnehme - von Gott nahtlos verschweißt wurden. 
These 1:

\section{In Moderne wie Vorgeschichte finden Menschen Dinge vor, die sie sich anzueignen versuchen.}

So betrachtet befand sich der Urmensch nicht in einer sehr viel komfortableren und andersartigen Ausgangssituation - einer paradiesischen Welt ohne Apple -, sondern in der sehr viel verzweifelteren Position einer Super-Apple-Welt mit einem echten iGod, aber ohne Schrauben und Kundenhotline. Umgekehrt mag gerade deshalb ein Blick darauf, wie sich der Urmensch diese seine Umwelt aneignen konnte, auch informativ für die Einschätzung unserer heutigen Situation sein. Damit komme ich schon zu meiner zweiten These:

\section{These 2:}

\section{Der Mensch eignete sich historisch seine Umwelt in einer Reihe von ,Zweckentfremdungen' (Exaptationen) an.}

Diese These entnehme ich aus der aktuellen Literatur des Forschungszweigs der sogenannten kognitiven Archäologie, wo sie zumindest vorsichtig angedacht wird. ${ }^{1}$ Der dort verwendete Terminus technicus für Zweckentfremdung lautet ,Exaptation'. Er wurde 1982 von den Biologen Stephen J. Gould und Elisabeth Vrba geprägt, um einen alternativen Mechanismus der biologischen Evolution neben der Adaption zu beschreiben: nicht evolutionäre Bewährung eines zufällig durch Mutation neu entstandenen Merkmals, sondern ,Recycling' eines bestehenden Merkmals in einem neuen Zusammenhang, wobei das Merkmal selbst durch Adaption zu einem anderen Zweck entstanden sein kann oder als ursprünglich funktionsloses Nebenprodukt einer solchen Adaption, d.h. als sogenannter ,Zwickel' oder ,Spandrille“. ${ }^{2}$ Gould und Vrba nennen als Beispiel das Federkleid des Archaeopteryx, welches als Adaption zur Thermoregulierung oder zur Insektenjagd entstanden sei und sich erst später als Fluginstrument entpuppt habe. Von „Recycling“ spricht der Neurowissenschaftler Stanislas Dehaene im Zusammenhang mit der Hypothese, dass das neuronale Modul zur Mustererkennung von den heutigen Menschen im Symbolgebrauch von Lesen und Schreiben ,zweckentfremdet' würde - auch dies ein klassischer Fall von Exaptation. ${ }^{3}$ Dieses letzte Beispiel weist bereits darauf hin, dass Exaptation auch für die Entwicklung des Menschen eine entscheidende Rolle gespielt hat. Man denke nur an die Bedeutung, die dem Sprechen für den Menschen zukommt, während Mund, Zähne und Lippen aber ursprünglich Instrumente der Nahrungsaufnahme sind. Der

1 Beaune 2008, Haidle 2006, d'Errico et al. 2017, aber schon Noiré 1880 und Hartig 1888.

2 Gould/Lewontin 1979.

3 Vgl. Dehaene 2004. 
Mund musste erst durch die Hand „befreit“ werden, um - zweckentfremdet - diese entscheidende Rolle spielen zu können. ${ }^{4}$

Exaptation ist nun nicht nur ein Mechanismus der biologischen Evolution, sondern auch in unserem Alltag omnipräsent - nämlich immer dann, wenn wir mit einem Artefakt improvisieren, etwa ein Messer als Schraubenzieher verwenden, einen Topf als Trommel, einen Aschenbecher als Briefbeschwerer, einen Regenschirm als Defensivwaffe und was einem sonst noch einfallen mag. Dass wir diesen kreativen Umgang mit Dingen in unserem Alltag tatsächlich pflegen, ist aber nicht die These der Archäologen und Anthropologen. Diese schlagen vielmehr vor, dass die Exaptation gleich ihrem biologischen Gegenstück einen wichtigen Mechanismus der kulturellen Evolution darstellt. Solche Fälle, in welchen die Zweckentfremdung nicht bloß als lokale Improvisation auftritt, sondern einen kulturellen Fortschritt darstellt, der sich in der Kultur verallgemeinert und verfestigt, kennen wir ebenfalls aus der Literatur. Der Ethnologe Lucien Lévy-Bruhl beschrieb bereits Anfang des zwanzigsten Jahrhunderts, wie in der Praxis des Zählens aus Namen von Körperteilen Zahlwörter werden können. ${ }^{5}$ Dieses ist ein schönes Beispiel, da es uns besonders gut vor Augen führt, dass der Mechanismus der Exaptation schon deshalb ein Schattendasein in der Literatur führt, da er unseren inhärent ,idealistischen ' Denkgewohnheiten zuwiderläuft. Wir sind es nämlich gewohnt, den Zwecken ein Primat gegenüber den Mitteln einzuräumen. Im Sprechen individuieren wir Handlungen meist nach ihren Zwecken, nicht nach den Mitteln (,Flaschenöffnen' statt ,Eindrehen und Ziehen des Korkenziehers', ,Kommunizieren“ statt ,Andere Ansprechen‘ usw.). Die Exaptation zwingt uns zur gegenteiligen Betrachtung: nicht sucht der gegebene, ideal fixierte Zweck seine materiellen Mittel, sondern das gegebene Mittel ,drängt' zu neuen Zwecken. ${ }^{6}$ LévyBruhl bringt diesen „paradoxen“ Aspekt sehr gut zum Ausdruck:

Kurz, so paradox diese Schlussfolgerung auch erscheinen mag, ist es gleichwohl der Fall, dass in den primitiven Gesellschaften der Mensch schon seit langen Jahrhunderten zählte, bevor er überhaupt Zahlen besaß. ${ }^{7}$

Der Fehler besteht in der Vorstellung, der „menschliche Geist“ habe sich die Zahlen geschaffen, um zu zählen, während in Wirklichkeit die Menschen ganz im Gegenteil zuerst zählen - mit Mühe und unter Verausgabung -, bevor sie die Zahlen als solche ersinnen. ${ }^{8}$

\footnotetext{
4 Leroi-Gourhan 1965, $162 \mathrm{f}$.

5 Lévy-Bruhl 1910, $204 \mathrm{ff}$.

6 Die Rede vom „Drängen“ ist dabei durchaus wörtlich gemeint. Die Anführungszeichen tragen nur der Tatsache Rechnung, dass es nicht ausreicht, eine solche Einwirkung des Werkzeugs auf seinen Verwender einfach zu postulieren, sondern auch der zugrundeliegende Mechanismus benannt werden muss. Die Theorie des „material engagement“ von Lambros Malafouris (2013) ist ein Schritt in diese Richtung.

7 Levy-Bruhl 1910, 232. Übersetzung O.S.

8 Levy-Bruhl 1910, 234. Übersetzung O.S.
} 
Dieser ,materialistische‘ Gegenstandpunkt, der die materiellen Mittel gegenüber den geistigen Zwecken betont, harmoniert auf natürliche Weise mit den Bedürfnissen der Archäologie. Denn zum einen sind Archäologen schon rein methodisch auf einen gewissen, nämlich methodologischen ,Materialismus“ verpflichtet, da eben nur die materiellen Mittel der frühen Menschen uns überkommen sind, während ihre geistige Kultur mit diesen Menschen vergangen ist. ${ }^{9}$ Zum anderen aber verspricht der Mechanismus der Exaptation einen echten explanatorischen Mehrwert in dem Versuch, die technische Geschichte der Menschheit zu verstehen. In der Frage nach der Entstehung neuer Techniken und Werkzeuge muss man nicht mehr auf den sprichwörtlichen, aber explanatorisch fragwürdigen ,Geistesblitz، setzen, sondern kann die Neuerung durch das „günstige Milieu“ erklären, in welchem sich allmählich die notwendigen strukturellen Bedingungen anreichern, bis die Neuerung die Akteure gleichsam anspringt und bloß noch von ihnen aufgegriffen werden muss. ${ }^{10}$ Die Archäologin Sophie A. de Beaune formulierte dementsprechend das explizite Programm, technische Neuerungen als „the result of the combination of preexisting elements rather than creations ex nihilo" zu begreifen. ${ }^{11}$

Exaptation nimmt damit Gestalt als (universeller?) Mechanismus der kulturellen Evolution an. Der Soziologe Émile Durkheim unterstrich, dass man bei sozialen Institutionen immer mit Exaptationen rechnen müsse. ${ }^{12}$ Wilhelm Wundt räumte ihm unter dem Titel der Heterogonie der Zwecke dann wenig später einen fundamentalen Platz als Grundlage „völkerpsychologischer“ (d.h. ethnologischer) Entwicklungsgesetze ein. ${ }^{13}$ Der Mechanismus der Exaptation beruht auf einem einfachen und plausiblen Gedanken: Historisch eignen sich die Menschen die Welt im Werkzeuggebrauch fortschreitend an, seien diese Werkzeuge nun materieller, symbolischer oder sozialer (institutioneller) Natur. Aber auch die im Artefakt angeeignete Natur ist nie zu einhundert Prozent kontrolliert, sondern behält eine Eigenständigkeit und Eigengesetzlichkeit,

9 Und so ist es auch kein Zufall, dass es (nicht bloß methodische, sondern philosophische) Materialisten waren, die die Bedeutung der Exaptation als historisches Entwicklungsprinzip erkannten und explizierten. So verallgemeinern Gideon Freudenthal und Peter McLaughlin ausgehend von einem ,exaptationalistischen 'Ansatz in der Wissenschaftshistoriographie: „This thesis may be generalized: the means available are decisive in conceptualizing a need. [...] Means are not developed in order to satisfy existing needs (or interests), but the concrete conception of needs, purposes which may explain action, depends on the means available, that are then used to satisfy them. To a certain extent then, the means available can determine the possibility or at least the reasonableness of certain needs, interests and desires.“ (Freudenthal/McLaughlin 2009, 7)

10 „milieu favorable“, Leroi-Gourhan 1965, 222f; ähnlich Noiré 1880, X.

11 De Beaune 2004, 149.

12 Durkheim 2007 [1894], Kap. V.1.

13 Wundt 1917, Bd.7, 389: ,jene Heterogonie der Zwecke, bei der die jeweils vorhandenen Motive Nebenerfolge hervorbringen, die sich im Verlauf der weiteren Entwicklung selbst in mit Bewußtsein erstrebte Zwecke und schließlich in die Hauptzwecke umwandeln können, gegenüber denen die ursprünglichen allmählich zurücktreten und zuletzt ganz verschwinden“. Siehe dazu Janich 2006. 
die in den Kulturwissenschaften oft als ,Materialität' bezeichnet wird. Diese Materialität ist der Springquell neuer Nützlichkeiten - und das heißt: Zwecke -, die am Artefakt erst sukzessive entdeckt werden. Die technischen Artefakte sind immer ,reicher als ihre ursprüngliche Bestimmung. Sie bergen immer auch neue Zwecke in sich, zu denen sie als Mittel entdeckt werden können, und die sich oft sogar als so erfolgreich erweisen, dass alte, ursprünglichere Zwecke verdrängt werden.

Damit wir in einem anspruchsvollen Sinne von einem Entwicklungsgesetzt sprechen können, ist natürlich wichtig, dass die Exaptation eine produktive Zweckentfremdung darstellen kann (wenn auch nicht muss), die mithin auf einer wohldefinierten Achse zu einem Fortschritt führt. Die Archäologin Miriam Haidle unterscheidet dementsprechend zwischen bloßer Abwandlung eines Verhaltens oder eine Körperfunktion einerseits und andererseits einem ,entirely new behavior, which requires the recognition of an unknown problem as well as new problem-solution relationships, new raw materials, new actions and new rewards". ${ }^{14}$ Als relevante Achse benennt Haidle eine Art Durchmesser des „kognitiven Raums“:

Every innovation in tool use does not have to represent a cognitive expansion. Innovation is when a known solution is implemented using new raw materials or when an existing solution is applied to a new problem. It can implement technological changes in production and application, but does not expand the cognitive aspect of the process. ${ }^{15}$

Der kognitive Raum ist eine komplexe Entität, die in der kognitiven Archäologie eine zentrale Rolle spielt. An dieser Stelle ist es aber nicht nötig, alle seine Dimensionen zu erkunden. Wir interessieren uns nur für eine Dimension, die in der Erfassung gänzlich neuer Zwecke besteht. Man kann sich dies an dem erwähnten Beispiel der Zahlwörter verdeutlichen. Lévy-Bruhl deutete bloß an, dass die aus der Exaptation der Namen von Körperteilen entstehenden Zahlen das Zählen erleichterten, also denselben Zweck auf einfacherem Wege zu erreichen erlaubten. Tatsächlich eröffnet ihre Erfindung aber die Möglichkeit, nicht nur zu zählen, sondern auch zu rechnen, was klar einen qualitativen Entwicklungsschritt hin zu neuen Zwecken darstellt. ${ }^{16}$

14 Haidle 2006, 182.

15 Haidle 2006, 282.

16 Der zu dieser kreativen Mittelaneignung - meist vermutlich durch eine neue Generation - komplementäre kulturelle Mechanismus ist der des - wie man sagen könnte - atavistischen Mittelgebrauchs, in welchem ein neues Mittel zwanghaft Normen unterworfen wird, die mit seinem Auftreten eigentlich obsolet sind. Michael Ott analysiert im vorliegenden Band den Buchdruck, der sich zuerst noch an den Normen der Handschrift orientiert. Ein anderes Beispiel wäre die Porträtphotographie, die, wie Gisèle Freund herausarbeitete (1968 [1936], insb. 76f.), sich den ästhetischen Normen der Malerei zumindest so, wie das neue, kleinbürgerliche Publikum diese versteht - unterwirft, statt konsequent die ihr inhärenten Möglichkeiten zu erkunden und entsprechende ästhetische Normen zu entwickeln (d.h. Normen, von denen man sagen könnte, dass sie sich mit der ihnen zugrundeliegenden Technik in einem „Gleichgewicht“ befinden, vgl. Hahn/Schlaudt 2016). Als aktuelles Beispiel könnte man die Fernsehberichterstattung nennen, welche, statt die Möglichkeiten des gegenüber dem Radio nun 
Damit sind wir aber wieder am Anfang unseres Gedankengangs angelangt. Wir haben gesehen, wie die Menschen historisch die schraubenlose Black Box öffnen: Sie erkunden sukzessive ihre Zweckmäßigkeiten und ,erschöpfen ' sie dabei in einem epistemisch substantiellen Sinne, ohne je eine Schraube lösen zu müssen. Dies will ich als dritte These behaupten:

These 3:

Zweckentfremdung spielt in der sukzessiven Aneignung von Naturgegenständen dieselbe Rolle, wie das reverse engineering bei modernen Artefakten.

Während man ein modernes Artefakt zu öffnen versuchen kann, um seine innere Natur zu erkunden, hat man bei einem Naturgegenstand diese Wahl nicht. Ein Stein (oder ein Stoßzahn, das Geweih eines Rentiers, ein Stück Holz, ein Klumpen Ton etc.) hat überhaupt kein „Inneres“ in einem qualitativ unterschiedenen Sinne, sondern besteht sozusagen nur aus einer in sich gefalteten Oberfläche: Bricht man den Stein auf, sieht man wieder nur eine Oberfläche. Die Menschen der Vorzeit sind somit angesichts der sie umgebenden Dinge gewissermaßen zum Behaviorimus verurteilt und haben keine andere Wahl, als das Äußere, nämlich die möglichen Verhaltensweisen, als das Innere zu nehmen und somit die innere Natur in der sukzessiven Erkundung dieser Möglichkeiten durch Exaptation nach außen zu stülpen.

Da Technikphilosophie, die wir hier betreiben, aber immer Epistemologie und Ethik zugleich ist, will ich dieser epistemologischen These auch eine ethische oder anthropologische Perspektive zur Seite stellen, auch wenn diese erst in den folgenden Abschnitten voll entfaltet wird. Welches sind nämlich die Implikationen des Exaptations-Ansatzes für unser Menschenbild? Es ist das Mindeste, $\mathrm{zu}$ sagen, dass diese Implikationen uneindeutig sind. Zum einen stellt der Ansatz der Exaptation einen klassischen Vertreter eines technologischen Determinismus dar. ,Materialität‘ der Artefakte bedeutet, dass eine Eigengesetzlichkeit der Artefakte in der dynamischen Entwicklung eingeräumt wird. Dieser Raum muss aber vom Spielraum des Menschen abgezogen werden. Wenn in einer Eigengesetzlichkeit des Artefakts technisch Mögliches auch zur Wirklichkeit drängt, geschieht dies über die Köpfe der Akteure hinweg. Einerseits. Andererseits aber können die Akteure den als Materialität beschriebenen Reichtum an Möglichkeiten zumindest subjektiv auch als Freiheit erleben, da die Materialität, solange sie nicht erschöpft ist, eben Alternativen bereithält zu dem, was vormals als richtig galt (diesen Aspekt werde ich in These 5 wieder aufgreifen). Diese Gedanken sind erst einmal so banal wie abstrakt, werden aber bald Substanz annehmen.

gegebenen Bildraums zu erkunden und zu nutzen, in diesem nur eine zusätzliche Bürde sieht: ein Raum, der nun neben der Tonspur noch zusätzlich zu füllen ist, oft mit irrelevantem Archivmaterial. 


\section{Das verlorene Paradies}

Wir springen nun von der Urzeit in die kapitalistische Moderne - ein gewaltiger Sprung, den aber der amerikanische Anthropologe Marshall Sahlins schon an unser statt vollführte, so dass ich mich im Folgenden auf seine Analyse beziehen kann. Verschaffen wir uns vorab einen ersten Eindruck von dem veränderten Verhältnis von Mensch und Werkzeug. Für die Vorgeschichte hielt Ludwig Noiré emphatisch fest: „Das Thier ist Sclave der Organe, der Mensch ist Herr der Werkzeuge.“17 Betreten wir hingegen mit Eugen Schmalenbach, Gründerfigur der Betriebswirtschaftslehre, einen Maschinenraum der 1920er Jahre, bietet sich uns ein ganz anderes Bild: „Zehn große Turbinen und Generatoren nebeneinander. Sie drehen, drehen, drehen sich, leise und schweigsam. Des helfenden Menschen bedürfen sie wenig. "18 Das Bild ist nicht dasselbe. In der Analyse der Veränderung können wir uns nun auf Sahlins stützen, der sorgfältig die Achse zu bestimmen versuchte, auf welcher die relevanten Verschiebungen zu vermerken sind:

On a very broad view of cultural evolution, technical developments have accumulated not so much in ingenuity as along a different axis of the man-tool relationship. It is a question of the distribution of energy, skill, and intelligence between the two. In the primitive relation of man to tool, the balance of these is in favor of man; with the inception of a 'machine age' the balance swings definitively in favor of the tool. [...] Typically, the instrument is an artificial extension of the person [...] But the latest technology would invert this relationship between man and tool. It becomes debatable which is the tool. ${ }^{19}$

Sahlins orientiert in sich in dieser Analyse sichtlich an dem sogenannten Maschinenfragment von Karl Marx, d. h. dem Kapitel „Fixes Kapital und Entwicklung der Produktivkräfte der Gesellschaft“ aus den Grundrissen der Kritik der politischen Ökonomie von 1857/58. „Fixes Kapital“ ist nichts anderes als die ökonomische Kategorie für die (als Investition betrachteten) materiellen Produktionsmittel, d. h. die Werkzeuge und Maschinen. Im Maschinenfragment analysierte Marx die besondere Gestalt, die die Maschine in der modernen Fabrik angenommen hat. Sie stellt dort keinen Einzelgegenstand in der Hand des Arbeitenden mehr dar, sondern hat sich zu einem ,automatischen System der Maschine“ weiterentwickelt:

In den Productionsprocess des Capitals aufgenommen, durchläuft das Arbeitsmittel aber verschiedne Metamorphosen, deren lezte die Maschine ist oder vielmehr ein automatisches System der Maschinerie (System der Maschinerie; das automatische ist nur die vollendetste adaequateste Form derselben und verwandelt die Maschinerie erst in ein System), in Bewegung gesezt durch einen Automaten, bewegende Kraft, die sich selbst bewegt; dieser Automat bestehend aus zahl-

17 Noiré 1880, 106.

18 Schmalenbach 1928, 244.

19 Sahlins 1972, 80. 
reichen mechanischen und intellectuellen Organen, so daß die Arbeiter selbst nur als bewußte Glieder desselben bestimmt sind. ${ }^{20}$

Marx beschreibt hier zum einen eine Entwicklungstrajektorie der Maschinentechnik, die sich im letzten Stadium zu einem autonomen, selbstbewegenden System schließt. Und zum anderen beginnt er bereits, die Konsequenzen für die Arbeiter zu benennen. Diesen Aspekt führt er im Anschluss weiter aus. Die zum System gewordene Maschine ist nicht mehr ein Werkzeug in der Hand des Arbeiters, sondern der Arbeiter wird zum Werkzeug in der Hand der Maschine:

Nicht wie beim Instrument, das der Arbeiter als Organ mit seinem eignen Geschick und Thätigkeit beseelt, und dessen Handhabung daher von seiner Virtuosität abhängt. Sondern die Maschine, die für den Arbeiter Geschick und Kraft besizt, ist selbst der Virtuose, die ihre eigne Seele besizt in den in ihr wirkenden mechanischen Gesetzen und zu ihrer beständigen Selbstbewegung, wie der Arbeiter Nahrungsmittel, so Kohlen, Oel etc consumirt (matières instrumentales). Die Thätigkeit des Arbeiters, auf eine blose Abstraction der Thätigkeit beschränkt, ist nach allen Seiten hin bestimmt und geregelt durch die Bewegung der Maschinerie, nicht umgekehrt. ${ }^{21}$

Marx stützt sich hier seinerseits offenkundig auf die von Kant in der Metaphysik der Sitten entwickelte Gedankenfigur der Um- oder Verkehrung (oder auch ,Perversion'), welche insbesondere auch den Vorwurf der Instrumentalisierung ausmacht: der (Selbst)Zweck wird zum (bloßen) Mittel verkehrt. ${ }^{22}$ Sahlins greift diese Gedankenfigur auf und sieht sich durch sie auch berechtigt, den Bogen zum Begriff der Entfremdung zu schlagen, die Marx bereits 1844 in den Ökonomisch-Philosophischen Manuskripten in der Tat auch als eine Mittel-Zweck-Verkehrung beschrieben hatte: Das Bewusstsein als Gattungsmerkmal des Menschen ist in der Lohnarbeit nicht mehr Selbstzweck, sondern wird zu einem bloßen Mittel degradiert. ${ }^{23}$ Dies gilt natürlich in besonderem Maße von der Arbeit in der automatisierten Fabrik, in welcher der Arbeiter bloß mehr als ,bewusstseinsbegabtes Zahnrad' die verbleibenden Lücken füllt, die noch nicht durch einen automatisierten Mechanismus geschlossen werden konnten. Hier macht der Mensch sein „Wesen nur zu einem Mittel für seine Existenz“. ${ }^{44}$ Sahlins verweist durch den Gebrauch des Wortes „alienated“ auf diesen Zusammenhang. Er erklärt gleichzeitig, welchen Aspekt er im Umschlag des Verhältnisses von Mensch und Werkzeug für entscheidend hält, nämlich den der „Entfremdung von den Fähigkeiten“, der Tatsache also, dass moderne Fabrikarbeit (gegenüber dem Handwerk) in gering-qualifizierter Arbeit besteht, die den Arbeiter seiner Fähigkeiten beraubt:

20 Marx/Engels (1975-2019), MEGA II/1.2, 571.

21 Marx/Engels (1975-2019), MEGA II/1.2, 572.

22 Kant 1903 [1785], 434f.

23 Marx/Engels (1975-2019), MEGA I.2, 367; vgl. auch Schlaudt 2019.

24 Marx/Engels (1975-2019), MEGA I.2, 369. 
For Marx, it should be noted, the critical turning point in the man-tool relation was not the substitution of nonhuman power, but the attachment of tools to a transmission and motor-mechanism; the last might still be human but the workman had effectively been alienated from the instruments of labor, the skill of handling them now passing over to the machine. ${ }^{25}$

Die wissenschaftliche Sorgfaltspflicht verlangt hier den philologischen Hinweis, dass der Bezug auf Marx an dieser Stelle auf wackeligen Füßen steht, da diese „Entfremdung von den skills“ in der klassischen Marxschen Entfremdungsanalyse der Ökonomisch-Philosophischen Manuskripte keine Rolle spielt. Dort heißt es nur, dass in der Lohnarbeit der Mensch das Mittel seiner Freiheit - die Arbeit - als Mittel seiner Knechtschaft erfahren muss - unabhängig davon, ob diese Arbeit qualifiziert oder unqualifiziert ist. Der von Sahlins herangezogene Zusammenhang wird am ehesten noch im Manifest der kommunistischen Partei ausgesprochen, in welchem umgekehrt die Entfremdung nicht dem Wortlaut nach auftaucht:

Die Arbeit der Proletarier hat durch die Ausdehnung der Maschinerie und die Teilung der Arbeit allen selbständigen Charakter und damit allen Reiz für die Arbeiter verloren. Er wird ein bloßes Zubehör der Maschine, von dem nur der einfachste, eintönigste, am leichtesten erlernbare Handgriff verlangt wird. ${ }^{26}$

Diese Zeitdiagnose, die wir hier probeweise von Sahlins übernehmen, gilt prima facie selbstverständlich nur mit einer wichtigen Einschränkung, welche der amerikanische Anthropologe selbst in einer Fußnote benennt: „Of course a great deal of knowledge is required for the development and maintenance of modern machinery; the above sentence confines itself to the relation of man and tool in the process of production." 27 Während mir der Verweis auf die technische Intelligenz, welche allein das Maschinensystem entwickeln und warten kann, durchaus triftig erscheint, halte ich die Beschränkung auf die Produktion für zu rigide. Die Diagnose eines Umschwungs von der Autonomie des Menschen, der das Artefakt als Werkzeug in der Hand hält, zur Autonomie der Maschine, welchen den Menschen als Werkzeug in der Hand hält, kann versuchsweise auf jeglichen Umgang mit der Technik ausgedehnt werden, d. h. sowohl auf die nicht-fabrikmäßige Produktion als auch auf den rein konsumierenden Technikgebrauch. Somit übernehme ich als vierte These die Diagnose von Marshall Sahlins:

\section{These 4:}

Im (produktiven wie konsumierenden) Gebrauch der modernen Technik kehrt sich das Verhältnis von Mensch und Technik auf der Achse von verkörperten Fähigkeiten zugunsten des Artefaktes um.

25 Sahlins 1972, 81, n. 28.

26 Marx/Engels (1958ff.), MEW 4/468f.

27 Sahlins 1972, 80. 
Diese Diagnose will ich im Folgenden weiter erkunden, und zwar ex negativo, indem wir uns anschauen, welche Freiheit im Gebrauch der modernen Technik noch besteht. Auch dazu kann ich mich auf vorfindliche Literatur stützen, in diesem Fall auf einige Feuilletons, die der Philosoph Alfred Sohn-Rethel (1899-1990) in den 1920er Jahren aus dem Herzen des anarchischen Widerstands gegen die technische Moderne schrieb, nämlich der Stadt Neapel. Wir dürfen diese leichten und humorvollen Feuilletons durchaus als ernstgemeinte philosophisch-soziologische Studien lesen, die methodisch der ,impressionistisch-literarischen' Empirie der frühen Frankfurter Schule verpflichtet sind, welche hier insbesondere den entfremdeten Blick des Touristen strategisch einsetzt. Dieser unverständige Blick erfasst treffsicher das Wesentliche. So heißt es in Das Ideal des Kaputten von 1926: „Technische Vorrichtungen sind in Neapel grundsätzlich kaputt: nur ausnahmsweise und dank eines befremdlichen Zufalls kommt auch Intaktes vor." ${ }^{28}$

Im Oberflächlichen, welches der Reisende je nach Gemütslage als folkloristische Grille, Rückständigkeit, oder auch Ärgernis wahrnehmen wird, offenbart sich in Wahrheit jedoch etwas sehr viel Grundsätzlicheres, ein fundamental andersartiges Verhältnis zur Technik:

\begin{abstract}
Aber nicht daß diese [technischen Vorrichtungen] nun darum, weil sie kaputt sind, etwa nicht funktionierten, sondern beim Neapolitaner fängt das Funktionieren gerade erst da an, wo etwas kaputt ist. [... F]ür ihn liegt vielmehr das Wesen der Technik im Funktionieren des Kaputten. [...] Das Intakte dagegen, das sozusagen von selber geht, ist ihm im Grunde unheimlich und suspekt, denn gerade weil es von selber geht, kann man letztlich nie wissen, wie und wohin es gehen wird. [... G]anz sicher ist er solcher Unwesen nie [...]..$^{29}$
\end{abstract}

Für beide Fälle - das Funktionale des Kaputten wie das Dysfunktionale des Intakten gibt Sohn-Rethel Beispiele. Halten wir uns einen Augenblick an dem ersten Fall auf, für den ich eine längere Schilderung zitiere:

[Der Neapolitaner] geht mit einem Motorboot aufs Meer, sogar bei heftigem Wind, in das wir kaum den Fuß zu setzen wagten. Und es geht zwar niemals, wie es gehen sollte, aber so oder so doch immer gut. Mit unerschütterlicher Selbstverständlichkeit bringt er es, drei Meter von den Klippen, an denen ihn die wilde Brandung zu zerschmettern droht, zum Beispiel fertig, den beschädigten Benzinbehälter, in den das Wasser eingedrungen ist, abzulassen und neu zu füllen, ohne daß der Motor aussetzt. Wenn nötig, kocht er gleichzeitig auf der Maschine noch Kaffee für die Fahrgäste. ${ }^{30}$

Ein komplementäres Beispiel für das Problem des Intakten lieferte der Autor in dem Text Eine Verkehrsstockung in der Via Chiaia:

28 Sohn-Rethel 1990, 33.

29 Sohn-Rethel 1990, $34 \mathrm{f}$.

30 Sohn-Rethel 1990, 34. 
Welches Verhältnis die Neapolitaner zu solchen technischen Wunderwerken wie Eisenbahnen haben, konnte ich erfahren, als ich einmal in Castellammare war, dem Kriegshafen von Neapel. Ich wollte mit der Bahn nach Neapel zurück und fragte den Stationsvorsteher, wo der Bahnsteig sei und wann die Eisenbahn nun abgehen würde. Das wußte er nicht. Da sah ich weiter hinten eine Bahn stehen und fragte: ,Ist sie das?' ,Nein - ich weiß nicht. ' Ich ging hin - und sie war es. Aber sie fuhr noch nicht. Da traf ich ihn wieder und sagte: ,Das ist sie aber doch!' Darauf er: ,Ja, was ist, wenn diese Dinge von selber gehen? Wie soll man wissen, wohin sie gehen und wann sie gehen? ${ }^{31}$

Der Eisenbahn gesellen sich noch schlimmere Dinge hinzu, welche nicht nur planmäßig funktionieren, sondern, ,wie die Elektrizität, nicht eigentlich kaputt zu machen sind und bei denen auch nicht einwandfrei festzustellen ist, ob sie wirklich von dieser Welt sind“. ${ }^{22}$ Solche Dinge verweist der Neapolitaner in das Sakrale als ihrem angemessenen Ort, z. B. in Form einer Osrambirne, die in der Kirche die Statue der Heiligen Maria erstrahlen lässt.

Die Dramatik dieser Analyse, die Sohn-Rethel ja mit dem (von uns später noch zu klärenden) Gebrauch der philosophischen Großgeschütze von ,Wesen` und ,Unwesen“ anzeigt, verstehen wir vielleicht besser, wenn wir sie mit einer anderen Beschreibung eines ähnlichen Ortes an der Peripherie der entwickelten Welt vergleichen. Ich beziehe mich auf eine Beobachtung, die Ivan Illich einige Jahrzehnte später machte:

I know a coastal area in South America where most people support themselves by fishing from small boats. The outboard motor is certainly the tool that has changed most dramatically the lives of these coastal fishermen. But in the area I have surveyed, half of all outboard motors that were purchased between 1945 and 1950 are still kept running by constant tinkering, while half the motors purchased in 1965 no longer run because they were not built to be repaired. Technological progress provides the majority of people with gadgets they cannot afford and deprives them of the simpler tools they need. ${ }^{33}$

Der methodische Kontrast zwischen beiden Autoren könnte freilich kaum größer sein, aber uns interessiert hier der inhaltliche Unterschied, der trotz der gemeinsamen Tendenz, in der modernen Technik ein (soziales) Problem zu diagnostizieren, zwischen ihnen bestehen bleibt. Ich will versuchen, den verbleibenden Unterschied deutlich werden zu lassen, indem ich ihn auf die Spitze treibe: Für Illich besteht das Problem der modernen Technik darin, dass sie sich nicht reparieren lässt, während Sohn-Rethel das Problem ganz im Gegenteil darin sieht, dass sie sich nicht „kaputt machen“ lässt.

31 Sohn-Rethel 1990, 13.

32 Sohn-Rethel 1990, 35f.

33 Illich 1971, 59. Das Zitat stammt aus einem Artikel Illichs, den die Saturday Review 1971 als Leitartikel anlässlich des Erscheinens von Illichs Buch Deschooling Society brachte. Der Artikel wurde in spätere Ausgaben des Buches aufgenommen und findet sich in deutscher Übersetzung auch in Illich 1978b. 
Warum aber kann Sohn-Rethel in diesem Zug, der an der Elektrizität am deutlichsten sichtbar wurde, ein Problem sehen? Nun, offenkundig ist dies der Fall, weil er den Verschleiß der Technik als die Bedingung ihrer wahren Aneignung versteht. Und nun lässt sich der Unterschied zwischen Illich und Sohn-Rethel nüchterner benennen: die ,wahre Aneignung besteht für jenen in der Fähigkeit, die Technik zu reparieren - also im Grunde in einem ,konservativen“, nämlich Zweck-bewahrenden Umgang -, während dieser im widerständigen Neapel auf einen anarchischen, improvisierenden und kreativen Umgang mit der Technik in Form prekärer, ausrangierter oder gar defekter Artefakte stößt. Nach der langen Vorbereitung in diesem Text gehört jetzt nur noch wenig Scharfsinn dazu, in Sohn-Rethels Beobachtungen schlicht und ergreifend Fälle von Exaptation zu erkennen: In den Papierkörben des Philosophischen Seminar brüten Hühner, das Souterrain der Kirche dient als Stall, die kühlere Gasse und gar der Eisenbahntunnel dienen als Schlafstätte in der Hitze des Hochsommers, der heißlaufende Schiffsmotor als Kochplatte für den Espresso, der ausrangierte Motor eines Motorrads wird zum Sahne-Schlagen eingesetzt, usw. usf. Diese Dinge bilden das neapolitanische „Glücksarsenal des Kaputten“. ${ }^{34}$ Schlussendlich schwingt sich Sohn-Rethel an einem letzten Beispiel zu einer abstrakten Benennung des Phänomens auf, wobei er eine treffsichere Definition von Exaptation avant la lettre liefert:

Der modernen Technik geht's hier wie jenem weltverlorenen Schienenpaar, welches einsam und verrostet am Monte Santo die Straßen hinunterläuft. Das Feldgeschrei der kühnen Pläne, zu denen es, man weiß nicht wann, hierher verschlagen wurde, ist längst verklungen und vergessen. Mit beispielloser Kraft des Funktionierens aber spritzt es den jubelnden Straßenkindern das Wasser, welches aus irgendeiner verirrten Leitung durch seine Rinnen fließt, zu seligem Ergötzen in den Mund, und die ganze Nachbarschaft erfreut sich dieser hochwillkommenen Quelle. So etwa vereinigen sich in dieser Stadt die kompliziertesten Zweckinstrumente der Technik zu einfachster, doch nie erträumter Verrichtung. Zu der unfreiwilligen Stiftung solchen Nutzens sind sie vollendet umgemodelt, zu ihren eigentlichen Zwecken versagen sie konsequent. ${ }^{35}$

Nachdem nun die Exaptation als technischer Kern des von Sohn-Rethel beschriebenen Phänomens benannt ist, bleibt die Frage nach seiner ethischen, politischen oder existentiellen Bedeutung zu stellen. Sohn-Rethel übersieht nicht, dass sich die neapolitanische Eigensinnigkeit im Umgang mit der Technik durchaus aus einem ,rückständigen` oder zumindest rückwärtsgewandten Humus nährt. Es ist vom „agrarischen Untergrund“ der Stadt die Rede in Ansehung der Gewohnheiten, Fähigkeiten und Anschauungen, die noch aus einer bäuerlichen Gesellschaft in das urbane Leben hinüberragen. ${ }^{36}$ Sohn-Rethel beschreibt ein Manufakturwesen, wie es für das 17. Jahrhundert typisch war, und schließt eindeutig: „Neapel war also keine Welt im

34 Sohn-Rethel 1990, 13.

35 Sohn-Rethel 1990, 38.

36 Sohn-Rethel 1990, 9. 
Aufbruch, sondern eine sehr alt verwurzelte Welt, mit Wurzeln, die tatsächlich noch bis in die Feudalität zurückgeführt werden können“. ${ }^{37}$

In dieser Rückständigkeit geht das Phänomen politisch freilich nicht auf. Bei der Lektüre springen einem im Zusammenhang mit der Exaptation Wörter wie „Freiheit“ und „Souveränität“ in die Augen. Die bäuerlich-feudalen Wurzeln nähren in Neapel eine Eigensinnigkeit, die in Konfrontation mit den Zwängen der Moderne - Max Webers „stahlhartem Gehäuse“ -, insbesondere des modernen Maschinenwesens, durchaus im Sinne der menschlichen Freiheit und somit nolens volens emanzipatorisch wirkt. In diesem Sinne kommentiert auch Françoise Willmann die aktuelle französische Übersetzung von Das Ideal des Kaputten:

\begin{abstract}
Was ,den' Neapolitaner interessiert, ist die als solche empfundene Meisterung in der direkten und erfinderischen Auseinandersetzung mit dem Gegebenen. Es ist nicht das Wesen des Neapolitaners, was Sohn-Rethel in seinem Text so gut erfasst, sondern genau das Ideal des Kaputten. Das Kaputte verkörpert ein Ideal - kein kantianisches Ideal im Sinne eines Horizonts oder Leitfadens unseres Handelns, sondern ein bedrohtes, in Abwicklung befindliches Ideal. Das Kaputte stellt den Handlungsspielraum (marge de manœuvre) dar, dessen der Einzelne noch habhaft werden kann, während sich die Teilung geistiger und körperlicher Arbeit bereits unaufhaltsam vertieft. Sohn-Rethel setzt den Neapolitaner, der sich weigert, von seinem Menschsein abgeschnitten zu werden, wieder in seine Rechte ein. Im Gegensatz zum Maschinenstürmer entscheidet er sich nicht gewollt für den bewussten Kampf, sondern eignet sich auf seine Weise das Werkzeug an und wendet es gegen eine Moderne, die im Begriff ist, ihn sowohl seines Körper als auch seines Geists zu berauben. ${ }^{38}$
\end{abstract}

Aus dieser Analyse kann ich die fünfte und letzte These dieses Aufsatzes direkt übernehmen:

\title{
These 5:
}

\section{Der Spielraum der Exaptation bildet den Spielraum (marge de manœuvre), welcher einem sich als frei empfindenden, menschlichen Handeln angesichts der Maschine verbleibt. ${ }^{39}$}

Auf dieser Grundlage lässt sich nun auch endlich einsehen, wie Sohn-Rethel vom „Funktionieren des Kaputten“ als dem „Wesen der Technik“ und umgekehrt von den unzerstörbaren Technologien als „Unwesen“ sprechen konnte. Was an diesen

\section{Sohn-Rethel 1990, 15.}

38 Métraux \& Willmann 2019, 113, Übersetzung O.S.

39 Hier konvergiert Sohn-Rethel tendenziell auch wieder mit Ivan Illich: „These minorities already see that they-and all autochthonous cultural life-are threatened by mega-tools which systematically expropriate the environmental conditions that foster individual and group autonomy. And so they quietly determine to fight for the usefulness of their bodies, memories, and skills. “(Illich 1978a, 38). Ähnlich betonte jüngst auch François Jullien den emanzipatorischen Wert der Exaptation, die die Entwicklung des Menschen nicht als Realisierung einer menschlichen Natur, sondern per „décrochage“, nämlich exaptativen Bruch mit dem jeweilig erreichten Stand konstituiert (Jullien 2016, 70). 
Redeweisen stört, ist die Tatsache, dass Sohn-Rethel hier scheinbar verschiedene Modi des bloßen Umgangs mit Technik - exaptativ oder nicht - in die Technik selbst projiziert. Aber erinnern wir uns, dass Technik angeeignete Natur ist und Exaptation Aneignung der Technik. In der Technologie, die sich nicht anarchisch zweckentfremden lässt und die keinen improvisierenden Umgang erlaubt, begegnen wir mithin strukturell demselben Phänomen wie in der entfremdeten Arbeit in der Marx'schen Analyse: Das Mittel der Freiheit wird zum Mittel der Knechtschaft. Angeeignete Natur widersetzt sich ihrer Aneignung. Dieses Verhältnis aber betrifft nicht ,bloß unseren Umgang mit Technik. Bestimmte Technologien - so die Diagnose Sohn-Rethels schließen einen Umgang aus, den ihr Dasein als Technik aber verlangt. Es kommt zu einem Widerspruch zu ihrem Wesen, wodurch diese Dinge „Unwesen“ werden. Das Gegenbild stellt die zweckentfremdete Technik dar, die - so paradox dies scheinen mag - gerade aufgrund der Zweckentfremdung in Einklang mit ihrem eigenen Wesen steht: „Die Technik beginnt vielmehr eigentlich erst da, wo der Mensch sein Veto gegen den feindlichen und verschlossenen Automatismus der Maschine einlegt und selber in ihre Welt springt."40

Wie Françoise Willmann zu Recht unterstreicht, steht dieses „Einspringen“ in die Welt der Technik im Kontrast nicht nur zum passiven Erdulden der Automatisierung, sondern auch zur bewussten Sabotage der Maschinenstürmer, die das „Wesen der Technik“ natürlich ebenso verfehlen muss, weil es sie sozusagen handgreiflich negiert. Eine solche Negation der Technik verbannt Sohn-Rethel literarisch auf indirekte Weise aus dem Bereich des Möglichen, dadurch nämlich, dass „Neapel“ erinnerlich im Schatten des „Vesuv“ liegt, von welchem tatsächlich auch ein weiterer Text SohnRethels handelt (Vesuvbesteigung 1926). Der Vesuv steht im Hintergrund der Analyse Sohn-Rethels. Er verkörpert die Welt der Natur, d. h. zum einen der Natur im Sinne der Naturgewalten als der absoluten Schranke der Naturbeherrschung, und zum anderen der Natur im Sinne der ewigen Kulisse im Kontrast zum wandelbaren menschlichen Treiben. Die Technik setzt sich von beiden Aspekten ab - sie beherrscht Naturkräfte, aber sie ist auch provisorisch. Hört sie auf, provisorisch zu sein, so hört sie auch auf, beherrschbar zu sein, hört also auf, im eigentlichen Sinne Technik zu sein, um wieder Natur zu werden.

40 Sohn-Rethel 1990, 36. Die Rede vom „verschlossenen Automatismus“ erinnert frappierend an das Maschinenfragment aus Marxens Grundrissen, die freilich erstmalig 1939-41 in der ersten MEGA in der Sowjetunion publiziert wurden. Man kann Sohn-Rethels Text also nicht als direkte Antwort auf das Maschinenfragment lesen. 


\section{Das wiedergewonnene Paradies?}

Als Hauptthesen der bisherigen Überlegungen fasse ich noch einmal zusammen, dass sich der Mensch in Vorgeschichte wie Moderne die Welt durch eine Reihe von Zweckentfremdungen aneignet (These 2) und die Möglichkeit solcher Zweckentfremdungen seinen verbleibenden Handlungsraum (marge de manœuvre) bestimmt (These 5). Für die Vorgeschichte können wir festhalten, dass ein solcher Raum immer bestehen blieb, was sich schon darin zeigt, dass die meisten Werkzeuge mehrere Funktionen hatten, wie sich an den charakteristischen Gebrauchsspuren ablesen lässt. ${ }^{41}$ These 4 legt nun nahe, dass dieser Handlungsraum schrumpft. Dies ist die ,Schicksalsfrage': Wieviel Freiheit bleibt dem Menschen in der technisierten Welt?

Diese Frage hat die Technikphilosophie schon immer umgetrieben, die aber in ihren Bemühungen um eine Antwort nicht sehr weit gekommen ist, sondern sich allzu oft in weltanschaulichem Geraune über die Schicksalhaftigkeit der modernen Technik verloren hat. ${ }^{42}$ Dieses Unvermögen mag auch damit zusammenhängen, dass die Frage in einem begrifflichen Rahmen aufgeworfen wurde, der auf der Opposition von Mensch und Technik fußte. Dieser Lesart will ich daher sogleich einen Riegel vorschieben. Der Mensch ist in einer Koevolution mit der Technik entstanden und muss daher in Einheit mit der Technik verstanden werden. ${ }^{43}$ Die Autoren, auf die ich mich bisher stützte, erlauben uns aber, die Frage auf eine andere Weise zu stellen. SohnRethel warnte uns ja nicht vor dem „Wesen“ der Technik, sondern ihrem „Unwesen“. Die Technik wird zu einem Problem, wenn sie aufhört, Technik zu sein, nämlich paradoxerweise - wenn sie „funktioniert“, nämlich über den Menschen hinweg und damit gegen ihn arbeitet. Auch Sahlins begriff in die Technik nicht als eine Gefahr, die von außen an uns herantritt. Er fragte vielmehr ganz nüchtern nach der „distribution of energy, skill, and intelligence between the two“. Diese Perspektive ist erhellend: Wir können Mensch und Technik als evolutionäre Einheit vielmehr denn als Opposition begreifen und gleichwohl verschiedene Gleichgewichtszustände innerhalb ihrer Einheit unterscheiden. Um die problematische Lesart, die auf der Opposition von Mensch und Technik basiert, von Vornherein auszuschließen, formulieren wir die ,Schicksalsfrage` etwas anders:

\footnotetext{
41 Vgl. de Beaune 2000, 137. Der Handlungsraum wächst im Altpaläolithikum anfangs sogar noch parallel zur Werkzeugentwicklung, was aber keinen Widerspruch darstellt, da in dieser Phase der Mensch die Vielfalt seiner manuellen Fertigkeiten überhaupt erst am Werkzeug entwickelt. Der älteste Faustkeil war somit nicht das universellste Werkzeug, da sein Benutzer noch über kein nennenswertes Repertoire differenzierter Handgriffe verfügte, die er mit diesem Werkzeug hätte ausüben können.

42 Man siehe die Vorträge von Jünger, Heidegger, Heisenberg usw. im Rahmen der Vortragsreihe der Bayerischen Akademie der Schönen Künste, 16.-20. November 1953, in: Bayerische Akademie der schönen Künste 1954.
}

43 Siehe z. B. Ambrose 2001. 
Schicksalsfrage:

\section{Wohin weist die evolutionäre Trajektorie des technischen Menschen,} insbesondere hinsichtlich des Parameters der marge de manceuvre?

Die Beantwortung dieser Frage hängt davon ab, wie wir die Diagnose einer unbeherrschbaren Technik - Marxens „System der Maschine“, Sohn-Rethels „Unwesen“ des „verschlossenen Automatismus“, Illichs „mega-tools“, Sahlins „Umkehr“ des Verhältnisses von Mensch und Werkzeug im „machine age“ - bewerten. Bisher habe ich sie ungeprüft übernommen. In einem letzten Schritt will ich aber das versäumte nachholen und nach ihrer genauen Tragweite fragen.

Zwei verschiedene Lesarten von der Diagnose „moderner Technik“ drängen sich auf, und der Leser mag die eine oder die andere von beiden bei der Lektüre der vorangegangenen Seiten auch schon im Hinterkopf gehabt haben. Die erste Lesart ist diejenige, die Sahlins schon mehr oder minder ausdrücklich befürwortete: Das Problem der modernen Technik ist wesentlich ein soziales Problem. Denn nur für den Arbeiter (oder Konsumenten), nicht aber den Ingenieur gilt die Diagnose der ,unbeherrschten Herrschaft im Artefakt. Diese Lesart bildet eine Tradition, die sich bis heute durchzieht. Als ein aktuelles Beispiel sei der Essay Maschinenwinter von Dietmar Dath zitiert, der mit folgenden Worten schließt:

Die Menschen haben zugelassen, daß die Maschinen, die ihnen zu dieser Abschaffung [von Herren und Knechten] verhelfen könnten, zu Naturwesen werden, deren Früchte man nicht ernten kann, weil sie keine mehr hervorbringen; wie schlafende Pflanzen im Winter. Die Menschen müssen ihre Maschinen befreien, damit die sich revanchieren können. ${ }^{44}$

Was Dath nicht sagt, sich im Kontext aber versteht: „Befreiung“ meint hier wohl Befreiung aus dem Privateigentum. Das Problem ist ein soziales. Daths emanzipatorischen Impetus muss man dabei übrigens nicht teilen. Man kann die soziale Lesart des Problems moderner Technik versuchsweise auch als ein Verteilungsproblem darstellen, welches verschiedene politische Lösungsansätze erlaubt: Gesamtgesellschaftlich bleibt der technische Handlungsspielraum bestehen, er ist bloß ungleich verteilt, nämlich durch eine technische Intelligenz monopolisiert, während Arbeiter und Konsumenten von dieser Freiheit weitgehend ausgeschlossen sind. So sieht dies beispielsweise Vilém Flusser, der in der Antizipation von unintendierten Folgen der Technik (welche die Exaptation ermöglichen) im technischen Design sogar einen echten Fortschritt erblickt, „denn wir sind dann nicht mehr Spielball unserer eigenen Instrumente, sondern zumindest teilweise bewusste Agenten. The things are no longer in the saddle, and they no longer fully ride us. ${ }^{* 45}$ Das Abwürgen der Exaptation interpretiert Flusser mithin als Kontrollgewinn, und die ungleiche Verteilung (die er in

44 Dath 2002, 131.

45 Flusser 2015 [1987], 2. 
der pauschalen Rede vom „wir“ freilich verschleiert) hält er offenbar für unproblematisch. Die Tragweite der Schicksalsfrage ist in dieser Lesart eher gering.

Aber diese Lesart ist nicht die einzig mögliche. Ich stelle ihr eine zweite gegenüber, nach welcher das Problem nicht rein sozial ist, sondern der Technik inhärent, unabhängig von Fragen der sozialen Organisation von Produktion, Distribution und Konsumtion. Diese Sichtweise kann beispielsweise durch den modernen Klassiker Die Existenzweise technischer Objekte von Gilbert Simondon aus dem Jahr 1959 exemplifiziert werden.

Simondon ist bekannt für die These, dass technische Artefakte sich auf einer autonomen $-d$. h. von den menschlichen Bedürfnissen, auf die sie eigentlich intrinsisch bezogen scheinen, unabhängigen -, quasi-evolutionären Trajektorie einer zunehmenden „Konkretisierung“ bewegen. Am Anfang steht das „abstrakte Objekt“ als das ,anfälligere“, da im Grunde kompliziertere Arrangement. ${ }^{46}$ Dieses Objekt ist noch „künstlich“:

Die wesentliche Künstlichkeit eines Objekts liegt in der Tatsache, dass der Mensch eingreifen muss, um die Existenz dieses Objekts aufrechtzuerhalten, indem er es gegen die natürliche Welt schützt und ihm einen existentiellen Sonderstatus gibt. ${ }^{47}$

Solche Objekte können sich aber „stabilisieren“, was vor allem geschieht, indem Sekundäreffekte in den Mechanismus eingebunden werden ${ }^{48}$ - ähnlich einem materiell geschlossenen Ökosystem, welches auch keine ,offenen Enden' aufweist und dadurch an Stabilität gegenüber äußeren Einflüssen gewinnt. ${ }^{49}$ Das Objekt wird auf diese Weise immer „konkreter“, „natürlicher“, aber auch immer mehr durch eine innere Notwendigkeit bestimmt: „In einem aktuellen Motor ist jedes wichtige Teil dergestalt durch wechselseitigen Energieaustausch mit den anderen verbunden, dass es nicht anders beschaffen sein kann, als es tatsächlich ist.“50

Wir erkennen hier den Dualismus von Kunst und Natur wieder, wie wir ihn schon bei Sohn-Rethel unter den Chiffren „Neapel“ und „Vesuv“ kennengelernt haben.

46 Simondon 2012, 24.

47 Simondon 2012, 43.

48 Simondon 2012, $29 \mathrm{ff}$.

49 Odum 1969, 265.

50 Simondon 2012 [1959], 20, Hervorh. O. S. Wir können denselben Gedanken versuchsweise in dem Vokabular des französischen Informationstheoretikers Abraham Moles ausdrücken, der vorschlug, technische Artefakte auf den beiden Achsen struktureller und funktionaler Komplexität zu beschreiben (s. Moles 1969 und Abb. 1 im Beitrag von Daniel Irrgang; eine präzise, operationalisierbare Definition dieser beiden Grundbegriffe bleibt Moles freilich schuldig). Es stellt sich sodann die Frage, ob eine strukturelle Komplexität, die die funktionale Komplexität übersteigt (also in ihrer Struktur noch funktional nicht ausgeschöpfte Möglichkeiten birgt), wirklich eine Ressource für Exaptationen darstellt, oder aber die kreative Mittelaneignung nicht gerade blockiert, wie es Moles' Beispiele nahelegen. Naturgegenstände und ideale Artefakte nach Simondon wären vermutlich in jedem Fall durch ein Gleichgewicht beider Komplexitätstypen gekennzeichnet. 
Simondon beschreibt den Übergang von Kunst zu Natur als einen Zwangsläufigen und Gesetzmäßigen. In der Perfektion wird das Artefakt wieder zum Naturding. Sohn-Rethel benannte die Konsequenzen für den Menschen: Bei einem Artefakt, welches „natürlich“ geworden ist, muss der Mensch nicht mehr eingreifen - aber er kann es auch nicht mehr, zumindest nicht in einem belangvollen Sinne. Selbstverständlich kann man auch den Verbrennungsmotor, an welchen Simondon denkt, noch zweckentfremden und ihn beispielsweise als Gewicht verwenden, um eine Leiche im Gewässer zu versenken. Aber es ist keine kreative Aneignung denkbar, die im Sinne des im ersten Teil dieses Artikels erwähnten technischen Entwicklungsgesetzes eine Verbesserung oder Weiterentwicklung darstellte, die es einem erlaubt, nicht bloß alte Zwecke auf unverhältnismäßige Weise zu realisieren (zum Versenken der Leiche hätte auch ein Stein genügt), sondern prinzipiell neue Zwecke zu erreichen. Wenn Simondon recht hat, versiegt diese Möglichkeit in der modernen Technik - und nun übrigens auch für die technische Intelligenz -, und das Paradies wäre ein für allemal verloren.

\section{Literaturverzeichnis}

Ambrose, Stanley H. (2001), „Paleolithic Technology and Human Evolution“, in: Science 291, 1748-1753.

Bayerische Akademie der schönen Künste (Hg.), Clemens Podewils (Redaktion) (1954), Die Künste im technischen Zeitalter (Gestalt und Gedanke 3), München.

Beaune, Sophie A. de (2000), Pour une Archéologie du geste. Broyer, moudre, piler, Paris.

Beaune, Sophie A. de (2004), „The Invention of Technology: Prehistory and Cognition“, in: Current Anthropology 45 (2), 139-162.

Beaune, Sophie A. de (2008), L'homme et l'outil. L'invention technique durant la Préhistoire, Paris.

Dath, Dietmar (2002), Maschinenwinter. Wissen Technik Sozialismus. Eine Streitschrift, Frankfurt a. M.

Dehaene, Stanislas (2004), „Evolution of human cortical circuits for reading and arithmetic: The 'neuronal recycling' hypothesis“, in: Stanislas Dehaene, J. R. Duhamel, M. Hauser u. G. Rizzolatti (Hgg.), From monkey brain to human brain, Cambridge (MA).

Durkheim, Émile (2007 [1895]), Les règles de la méthode sociologique, Paris.

d'Errico, Francesco/Doyon, Luc/Colagé, Ivan/Queffelec, Alain/le Vraux, Emma/Giacobini, Giacomo/ Vandermeersch, Bernard/Maureille, Bruno (2017), „From Number Sense to Number Symbols. An Archaeological Perspective“, in: Philosophical Transactions of the Royal Society B 373 (20160518), 1-10.

Flusser, Vilém (2015 [1989]), „Rueckschlag der Werkzeuge auf das Bewusstsein“ (Typoskript von 1989, als Faksimile veröffentlicht), in: Graham Harman (2015), Die Rache der Oberflächlichkeit: Heidegger, McLuhan, Greenber (International Flusser Lecture), Köln.

Freudenthal, Gideon/McLaughlin, Peter (2009), „Classical Marxist Historiography of Science: The Hessen-Grossmann-Thesis“, in: Gideon Freudenthal u. Peter McLaughlin (Hgg.), The Social and Economic Roots of the Scientific Revolution. Texts by Boris Hessen and Henryk Grossmann (Boston Studies in the Philosophy of Science 278), Berlin, 1-40.

Freund, Gisèle (1968 [1936]), Photographie und bürgerliche Gesellschaft. Eine kunstsoziologische Studie (Erstdruck des Originaltextes; zuerst in französischer Übersetzung 1936), München. - dies. (1936), La Photographie en France au dix-neuvième siècle, Paris. 
Gould, Stephen J./Lewontin, R. C. (1979), „The Spandrels of San Marco and the Panglossian Paradigm: A Critique of the Adaptationist Programme“, in: Proceedings of the Royal Society of London. Series B, Biological Sciences 205 (1161), 581-598.

Gould, Stephen J./Vrba, Elisabeth S. (1982), „Exaptation - A missing term in the Science of Form“, in: Paleobilogy 8 (1), 4-15.

Hahn, Susanne/Schlaudt, Oliver (Hgg.) (2016), Logic, Morals, Measurement. Origins and Justification of Norms (= Analyse und Kritik 38 (2) [Sonderheft]), Berlin/Boston.

Haidle, Miriam Noël (2006), How to think tools? A comparison of cognitive aspects in tool behavior of animals and during human evolution (Cognitive perspectives in tool behaviour 1), Tübingen. Online-Publikation: http://nbn-resolving.de/urn:nbn:de:bsz:21-opus-60146 (Stand: 18.12.2019).

Hartig, Ernst (1888), „Ueber den Gebrauchswechsel des Werkzeuges und das gegenseitige Verhältniss verbaler und substantivischer Begriffe in der mechanischen Technik“, in: Der Civilingenieur 34 (8).

Illich, Ivan (1971), „The Alternative to Schooling“, in: Saturday Review, June 19, 44-48 und 59-60. Illich, Ivan (1978a), The Right to Useful Unemployment And Its Professionel Enemies, London. Illich, Ivan (1978b), Fortschrittsmythen, Reinbek b. H.

Janich, Peter, (2006), „Die Heterogonie der Zwecke als Problem der Psychologie“, in: G. Jüttemann (Hg.), Wilhelm Wundts anderes Erbe. Ein Missverständnis löst sich auf, Göttingen.

Jullien, François (2016), Il n'y a pas d'identité culturelle, Paris.

Kant, Immanuel (1903 [1785]), Grundlegung zur Metaphysik der Sitten (Kant's gesammelte Schriften, hg. von der Königl. Preuß. Akademie der Wissenschaften. Erste Abteilung: Werke, Bd. 4), Berlin. Leroi-Gourhan, André (1965), Le geste et la parole. Bd. 1: Technique et langage, Paris.

Lévy-Bruhl, Lucien (1910), Les fonctions mentales dans les sociétés inférieures, Paris. Malafouris, Lambros (2013), How things shape the mind, Cambridge (MA).

Marx, Karl/Engels, Friedrich (1958ff.), Werke (MEW), Berlin.

Marx, Karl/Engels, Friedrich (1975-2019), Gesamtausgabe (MEGA), hg. von der Internationalen Marx-Engels-Stiftung Amsterdam, Berlin.

Métraux, Alexandre/Willmann, Françoise (2019), „Postface“, in: Walter Benjamin, Asja Lacis u. Alfred Sohn-Rethel, Sur Naples, Bordeaux, 89-116.

Moles, Abraham A. (1969), „Théorie de la complexité et civilisation industrielle“, in: Communications 13, 51-63.

Noiré, Ludwig (1880), Das Werkzeug und seine Bedeutung für die Entwickelungsgeschichte der Menschheit, Mainz.

Odum, Eugene (1969), „The Strategy of Ecosystem Development“, in: Science 164, 262-270.

Sahlins, Marshall (1972), Stone Age Economics, New York.

Schlaudt, Oliver (2019), „Menschwerdung oder Entfremdung? Das Thema der Arbeit beim jungen Marx“, in: Fromm Forum 23, 187-206.

Schmalenbach, E. (1928), „Die Betriebswirtschaftslehre an der Schwelle der neuen Wirtschaftsverfassung “, in: Zeitschrift für Handelswissenschaftliche Forschung 22 (6), 241-251.

Simondon, Gilbert (2012 [1959]), Die Existenzweise technischer Objekte, aus dem Französischen von Michael Cuntz, Zürich.

Sohn-Rethel, Alfred (1990), Das Ideal des Kaputten, hg. von Carl Freytag, Bremen.

Wundt, Wilhelm (1917), Völkerpsychologie. Eine Untersuchung der Entwicklungsgesetze von Sprache, Mythus und Sitte, Bd.7 u. 8: Die Gesellschaft, Leipzig.

Zweig, Stefan (1944), Amerigo. Die Geschichte eines historischen Irrtums, Stockholm. 
